\author{
Birgit Simon \\ Stephan Schmidt \\ Carsten Lukas \\ Jürgen Gieseke \\ Frank Träber \\ Dirk L. Knol \\ Winfried A. Willinek \\ Jeroen J. G. Geurts \\ Hans H. Schild \\ Frederik Barkhof \\ Mike P. Wattjes
}

\section{Improved in vivo detection of cortical lesions in multiple sclerosis using double inversion recovery MR imaging at 3 Tesla}

Received: 15 September 2009

Revised: 5 November 2009

Accepted: 27 November 2009

Published online: 22 January 2010

(C) The Author(s) 2010.

This article is published with open access at Springerlink.com

B. Simon · J. Gieseke $\cdot$ F. Träber .

W. A. Willinek - H. H. Schild

Department of Radiology,

University Hospital of Bonn,

Bonn, Germany

\section{S. Schmidt}

Neurologische Gemeinschaftspraxis

Bonn,

Kölnstraße 480,

Bonn, Germany

\section{Lukas}

Department of Diagnostic and Interventional Radiology,

St. Josef Hospital,

University of Bochum,

Bochum, Germany

C. Lukas · J. J. G. Geurts ·

F. Barkhof - M. P. Wattjes $(\bowtie)$

Department of Radiology,

MS Center Amsterdam,

VU University Medical Center,

De Boelelaan 1117,

1081 HV Amsterdam, The Netherlands

e-mail: m.wattjes@vumc.nl

Tel.: +31-20-4444594

Fax: +31-20-4440397

\section{J. Gieseke}

Philips Medical Systems,

Hamburg, Germany

D. L. Knol

Department of Epidemiology

and Biostatistics,

VU University Medical Center,

Amsterdam, The Netherlands

\section{J. J. G. Geurts}

Department of Pathology,

VU University Medical Center,

Amsterdam, The Netherlands

\begin{abstract}
Objective: To investigate the impact of a higher magnetic field strength of 3 Tesla (T) on the detection rate of cortical lesions in multiple sclerosis (MS) patients, in particular using a dedicated double inversion recovery (DIR) pulse sequence. Methods: Thirty-four patients with clinically isolated syndromes or definite MS were included. All patients underwent magnetic resonance imaging (MRI) at $1.5 \mathrm{~T}$ and $3 \mathrm{~T}$, including $\mathrm{T} 2$-weighted turbo spin echo (TSE), fluid-attenuated inversion recovery (FLAIR) and DIR sequences. All images were analysed
\end{abstract}

for focal lesions categorised according to their anatomical location. Results: The total number of detected lesions was higher at $3 \mathrm{~T}$ across all pulse sequences. We observed significantly higher numbers of lesions involving the cortex at $3 \mathrm{~T}$ using a DIR sequence. DIR at $3 \mathrm{~T}$ showed $192 \%$ more pure intracortical $(p<0.001)$ and $30 \%$ more mixed grey matter-white matter lesions $(p=0.008)$. No significant increase in cortical lesions could be detected on the FLAIR and T2-weighted images. Using the T2-weighted and FLAIR sequences, significantly more lesions could be detected at $3 \mathrm{~T}$ in the infratentorial, periventricular and juxtacortical white matter.

Conclusion: DIR brain MR imaging at $3 \mathrm{~T}$ substantially improves the sensitivity of the detection of cortical lesions compared with the standard magnetic field strength of $1.5 \mathrm{~T}$.

Keywords Multiple sclerosis . Magnetic resonance imaging . Double inversion recovery . Cortical lesions $\cdot$ Pulse sequences

\section{Introduction}

Multiple sclerosis (MS) is the most common chronic inflammatory disease of the central nervous system (CNS) in young adults and is characterised by the presence of multifocal inflammatory demyelinating lesions disseminated in space and in time $[1,2]$. Although MS has been considered a disease primarily affecting the white matter, it has been conclusively shown that MS lesions occur abundantly in the grey matter as well $[2,3]$.

Magnetic resonance imaging (MRI) is the most sensitive paraclinical method in the detection of focal and diffuse abnormalities in the brain of MS patients. Using conventional MRI techniques, MS lesions are frequently and 
typically detected in certain anatomical regions, such as the periventricular, juxtacortical and infratentorial white matter, which is reflected by current MRI and clinical diagnostic criteria [1, 2, 4-7]. However, despite the histopathological abundance of cortical lesions in MS patients, the sensitivity of MRI in the detection of cortical lesions remains rather low $[3,8]$.

Because of the substantial increase in the signal-to-noise ratio (SNR), high-field MRI beyond 1.5 Tesla (T) offers several advantages and new possibilities especially in the field of neuroimaging [9]. In patients with suspected and/or definite MS, high-field MRI provides higher lesion load measurements of acute and chronic white matter lesions. This higher sensitivity becomes specifically obvious in anatomical regions that are important for the diagnosis and prognosis of MS [10]. However, whether higher magnetic field strengths also provide an improved in vivo detection of cortical abnormalities has not been investigated and remains questionable.

The aim of our study was to investigate the impact of a higher magnetic field strength of $3 \mathrm{~T}$ on the detection rate of cortical lesions in MS patients, in particular using a dedicated double inversion recovery (DIR) pulse sequence.

\section{Materials and methods}

Study design

This study was designed as an intraindividual comparative study in patients with a clinically isolated syndrome (CIS) suggestive of MS or patients with definite MS. The study protocol was approved by the local institutional review board and written informed consent was obtained from all participants. Inclusion criteria were: CIS of the central nervous system (CNS) suggestive of MS or definite MS as defined by the International Panel on MS diagnosis [2]; exclusion criteria were: any vascular, malignant and other immunological CNS diseases in the present and/or past medical history.

Patients and healthy controls

Thirty-four patients ( 26 female; median age at the time of MR examination 38 years, range 22-52 years) and nine healthy controls (three male, six female; median age at the time of MR examination 34 years, range 24-61.5 years) were included in this study. Two patients presented with a CIS and 32 patients had a relapsing-remitting course of MS (RR-MS) according to the revised International Panel criteria on MS diagnosis [2]. None of the patients received any treatment with corticosteroids in the month before the MR examination. All patients with RR-MS were under immune-modifying treatment at the time of the MR examination. The median disease duration (estimated from the first clinical event) of the CIS patients was 6.5 months (patient 1, 3 months; patient 2, 10 months), and 8 years (range 2 months to 26.3 years) for the RR-MS group. The median disability score of the patients according to the Expanded Disability Status Scale [11] at the time of the MRI examination was 1.6 (range $0-4$ ), indicating mild disability.

\section{MRI}

The MR examination was performed on 1.5-T and 3-T whole-body MRI (Achieva, Philips Medical Systems, Best, the Netherlands). The examinations at both field strengths

Table 1 Sequence parameters used at $1.5 \mathrm{~T}$ and $3 \mathrm{~T}$ (TR repetition time, $T E$ echo time, $I R$ inversion recovery, SENSE sensitivity encoding, NSA numbers of signals averaged)

\begin{tabular}{|c|c|c|c|c|c|c|}
\hline $\begin{array}{l}\text { System } \\
\text { Parameter }\end{array}$ & $\begin{array}{l}1.5 \mathrm{~T} \\
\text { FLAIR }\end{array}$ & $3 \mathrm{~T}$ & $\begin{array}{l}1.5 \mathrm{~T} \\
\mathrm{~T} 2 \mathrm{TSE}\end{array}$ & $3 \mathrm{~T}$ & $\begin{array}{l}1.5 \mathrm{~T} \\
\mathrm{DIR}\end{array}$ & $3 \mathrm{~T}$ \\
\hline Orientation & Transverse & Transverse & Transverse & Transverse & Transverse & Transverse \\
\hline Number of sections & 40 & 40 & 40 & 40 & 40 & 40 \\
\hline Slice thickness/gap (mm) & $3 / 0$ & $3 / 0$ & $3 / 0$ & $3 / 0$ & $3 / 0$ & $3 / 0$ \\
\hline Measured voxel size (mm) & $1 / 1 / 3$ & $1 / 1 / 3$ & $1 / 1 / 3$ & $1 / 1 / 3$ & $1 / 1 / 3$ & $1 / 1 / 3$ \\
\hline Echo train & 21 & 38 & 18 & 40 & 16 & 13 \\
\hline $\mathrm{TR}(\mathrm{ms})$ & 6,000 & 12,000 & 4,100 & 5,100 & 13,092 & 11,000 \\
\hline $\mathrm{TE}(\mathrm{ms})$ & 140 & 140 & 100 & 100 & 25 & 25 \\
\hline IR delay (ms) & 2,000 & 2,850 & - & - & $3,400 / 325^{\mathrm{a}}$ & $3,400 / 325^{\mathrm{a}}$ \\
\hline SENSE factor & 1.9 & 1.35 & 1 & 1 & - & 2 \\
\hline NSA & 2 & 1 & 2 & 1 & 2 & 2 \\
\hline Imaging time (min:s) & $5: 12$ & $4: 00$ & $3: 41$ & $1: 42$ & $8: 17$ & $6: 36$ \\
\hline
\end{tabular}

${ }^{\mathrm{a}}$ The long inversion time $\mathrm{TI}_{1}(3,400 \mathrm{~ms})$ is defined as the interval between the first $180^{\circ}$ inversion pulse and the $90^{\circ}$ excitation pulse. The short inversion time $\mathrm{TI}_{2}(325 \mathrm{~ms})$ is defined as the interval between the second $180^{\circ}$ inversion pulse and the $90^{\circ}$ excitation pulse 
were performed in a randomised order within a time interval of $12 \mathrm{~h}$. Both MRIs were equipped with standard gradients (a maximum slew rate of $150 \mathrm{mT} / \mathrm{m} / \mathrm{ms}$ and a maximum strength of $30 \mathrm{mT} / \mathrm{m}$ ). For the examinations at both MRI systems, an eight-element phased array sensitivity encoding (SENSE) head-coil (Philips Medical Systems, Best, the Netherlands) was used.

The imaging protocol at both field strengths included 40 contiguous sections in two-dimensional (2D) transverse orientation of the following pulse sequences: T2-weighted turbo spin echo (TSE), fluid-attenuated inversion recovery (FLAIR) and DIR. The MR sequence parameters were separately optimised for $1.5 \mathrm{~T}$ and $3 \mathrm{~T}$. The parameters are given in detail in Table 1. The same repositioning was used for all pulse sequences across both 1.5-T and 3-T MRIs according to the Consortium of MS Centers consensus guidelines [12].

\section{Image analysis}

The image analysis was performed by two radiologists in consensus, each with experience of at least 5 years in neuroradiology and with special expertise in the field of inflammatory demyelinating diseases such as MS. The images were analysed at a digital workstation (Centricity Radiology RA 600 version 6.1, General Electrics Medical Systems, USA) and the order of analysis randomised for the different pulse sequences of the MR examinations. Both readers were blinded to the clinical presentation and paraclinical tests (visual evoked potentials, CSF analysis, blood tests). The images of healthy controls were evaluated in terms of artefacts that might impair the diagnostic quality and lead to false-positive or -negative results. All images obtained from the patient group were analysed for focal lesions, which were categorised according to their anatomical location in: pure cortical, mixed white matter-grey matter, deep grey matter, juxtacortical, deep white matter, periventricular white matter and infratentorial lesions. Regarding the white matter lesions, we considered focal high signal intensity lesions with a size of $\geq 3 \mathrm{~mm}$. The classification in terms of different anatomical locations was performed according to published criteria $[4,5]$. Intracortical lesions and mixed white matter-grey matter lesions were defined as focal lesions with higher signal intensity than the normal appearing grey matter. Intracortical lesions were further defined as lesions purely located in the cortex not involving the white matter. Abnormalities only involving the subpial layer of the cortex suggesting subpial demyelination were not considered. White matter-grey matter lesions were defined as lesions partially located in the white matter as well as in the cortical grey matter. The criteria for the identification and classification of cortical lesions were adapted from recently published studies $[3,8$, $13,14]$. In order to avoid false positive results due to artefacts (in particular on the DIR), we discriminated between lesions and artefacts by considering images obtained from healthy control subjects.

\section{Statistical methods}

The relative comparison of numbers of lesions seen on $3 \mathrm{~T}$ versus $1.5 \mathrm{~T}$ for each type of sequence and separated for different anatomical locations was expressed as a percentage of the lesion number seen on 1.5-T MRI. A positive relative comparison indicates an increase in lesions identified at $3 \mathrm{~T}$ (higher sensitivity), whereas a negative relative comparison indicates a decrease in the number of detected lesions at $3 \mathrm{~T}$ (lower sensitivity).

The statistical evaluation of the patient-based analysis in terms of lesion load measurements (number of lesions) on $3 \mathrm{~T}$ and $1.5 \mathrm{~T}$, respectively, was performed with the Wilcoxon test for matched pairs. In order to account for clustering (repeated measurement) a negative binomial regression model with robust sandwich estimators was performed. In the case of a significant interaction between method (different pulse sequences: T2-weighted TSE, FLAIR, DIR) and technique (different magnetic field strengths: $1.5 \mathrm{~T}, 3 \mathrm{~T}$ ) the differences between the techniques were tested and adjusted for multiple comparisons (Bonferroni $p$ values).

$P$ values $<0.05$ were considered statistically significant. The data were analysed using SPSS version 17.0 (SPSS, Chicago, Ill., USA) and Stata version 10.0 (Stata, College Station, Tex., USA) for the negative binominal model.

\section{Results}

Healthy control subjects

\section{Focal lesions, artefacts}

The MR examinations of the eight healthy controls at both field strengths showed 13, 17 and nine lesions on the T2weighted TSE, FLAIR and DIR respectively. Most of the lesions were located in the deep white matter $[11(85 \%)$ on T2-weighted TSE, ten $(59 \%)$ on FLAIR, six $(67 \%)$ on DIR]. The remaining lesions were located in the periventricular white matter. Cortical lesions could not be identified on any of the used pulse sequences in any control subject.

DIR sequences of the healthy control subjects at $1.5 \mathrm{~T}$ and $3 \mathrm{~T}$ showed comparably high signal changes of certain cortical areas particularly involving the archicortex and palaeocortex as previously described $[13,15]$. Moreover, artefacts due to vessel and CSF pulsation could be observed more frequently and prominently on DIR sequences at both field strengths compared with FLAIR and T2-weighted TSE. These artefacts were particularly located in the posterior fossa close to the fourth ventricle, around the 
mesencephalic aqueduct and close to the choroid plexus (Fig. 1).

\section{MS and CIS patients}

All included patients (CIS and MS) showed focal lesions on MRI. The numbers of detected lesions at both field strengths separated according to their anatomical locations are listed in Table 2. The data of a patient-based analysis are presented in Table 3. The total number of lesions detected was higher at $3 \mathrm{~T}$ compared with $1.5 \mathrm{~T}$ across all pulse sequences. Separated by different anatomical locations, the lesion load at $3 \mathrm{~T}$ was significantly higher in the periventricular $(p=0.016)$ and deep white matter regions $(p=0.002)$ for the T2 weighted TSE, and in the infratentorial $(p=0.012)$, periventricular $(p=0.027)$, juxtacortical $(p=0.005)$ and deep white matter regions $(p<0.001)$ for the FLAIR sequence. For the DIR sequence, the higher lesion count at $3 \mathrm{~T}$ reached statistical significance for the infratentorial $(p<0.001)$, juxtacortical $(p=0.001)$ and deep white matter region $(p<0.001)$, but also regarding the pure intracortical $(p<0.001)$ and mixed grey matter-white matter lesions $(p=0.008)$ (Figs. 1, 2, 3 and 4).

The negative binomial regression model showed no significant interaction between the magnetic field strength and pulse sequences regarding the total number of lesions $(p=0.067)$. Considering the subgroup analysis for different anatomical locations, a positive interaction between the magnetic field strength and pulse sequence was significant for intracortical $(p=0.004)$ and infratentorial lesions $(p<$ $0.0001)$. Separated by the different pulse sequences, it became obvious that this interaction was driven by a significantly increased detection rate for FLAIR $(p=$ 0.0026; adjusted for multiple comparisons, $p=0.0078$ ) and DIR $(p<0.0001$; adjusted for multiple comparisons, $p<0.0001)$ but not for T2 TSE $(p=1)$ regarding infratentorial lesions. Concerning intracortical lesions this effect was only significant for the DIR $(p<0.0001$; adjusted for multiple comparisons, $p<0.0001$ ) but not for the FLAIR $(p=0.40$; adjusted for multiple comparisons, $p=1)$ and T2 TSE sequences ( $p=0.49$; adjusted for multiple comparisons, $p=1$ ).

\section{Discussion}

Since gaining the approval of the American Food and Drug Administration (FDA) in 2002, the advantages and challenges of brain imaging using clinical high-field MRI at $3 \mathrm{~T}$ have been extensively investigated. In patients with CIS suggestive of MS and definite MS, it has been conclusively shown that imaging at higher magnetic field strengths leads to significantly higher lesion load measurements [16-18]. This is particularly obvious in anatomical regions that are highly important for the diagnosis of MS in the context of diagnostic criteria and prognostic purposes such as the juxtacortical, periventricular and infratentorial white matter [10, 16-18]. Contrary to these studies, which were performed with a rather low spatial resolution using a slice thickness of $5 \mathrm{~mm}$, we used a higher spatial resolution

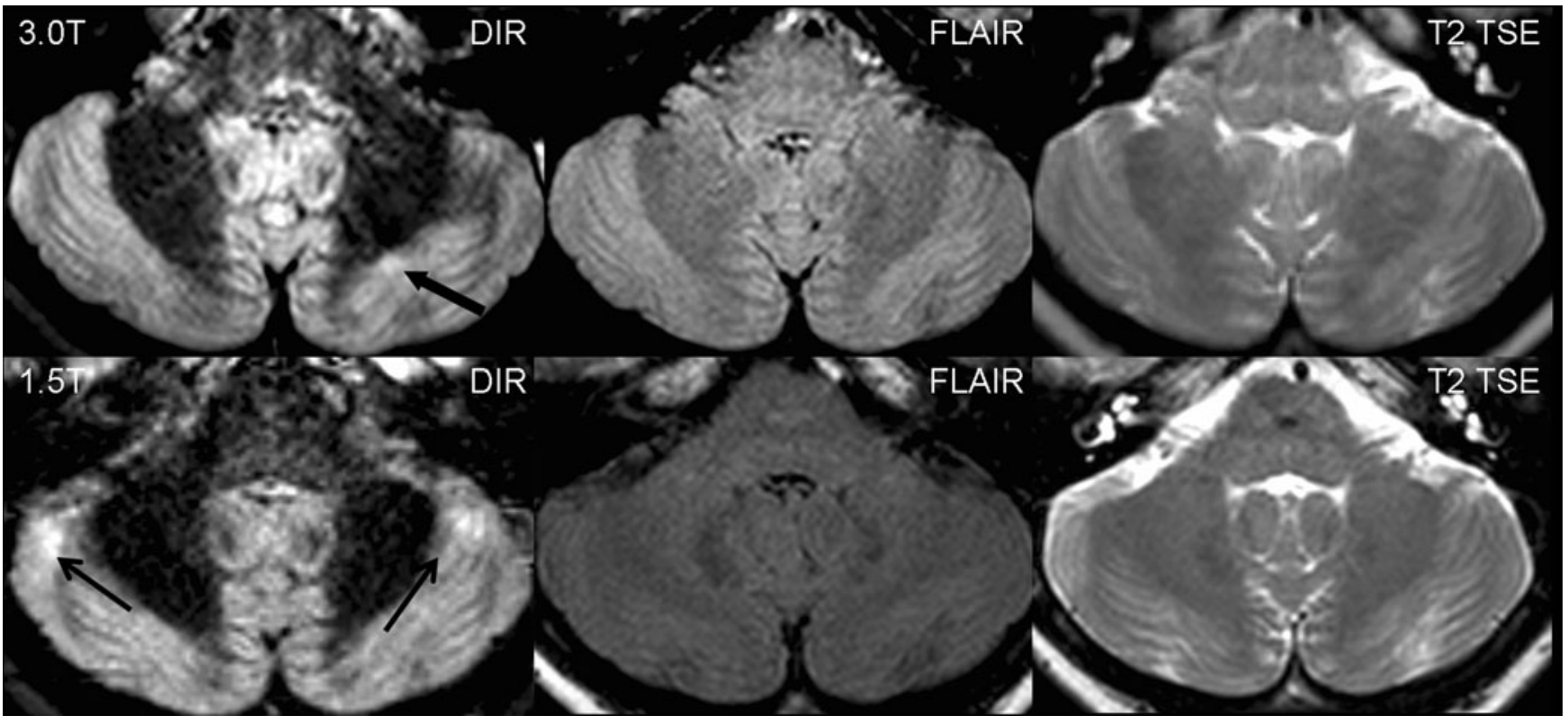

Fig. 1 Transverse sections of the infratentorial brain at $3 \mathrm{~T}$ (top row) and $1.5 \mathrm{~T}$ (bottom row) obtained from a 50-year-old woman with relapsing-remitting MS. The DIR sequence (left column) is able to detect more infratentorial lesions at $3 \mathrm{~T}$ compared with $1.5 \mathrm{~T}$ and other pulse sequences such as FLAIR (middle column) and T2 TSE (right column). Those lesions can also affect the cortex (closed arrowhead). Please note the higher propensity of DIR to vessel and CSF pulsation artefacts particularly in the posterior fossa (open arrowhead) 
Table 2 Numbers of lesions detected at $1.5 \mathrm{~T}$ and $3 \mathrm{~T}$ categorised according to different anatomical regions ( $G M$ grey matter, $W M$ white matter)

\begin{tabular}{|c|c|c|c|c|c|c|c|c|c|}
\hline \multirow[b]{2}{*}{ Region } & \multicolumn{2}{|l|}{$\mathrm{T} 2$} & \multirow[b]{2}{*}{ Relative comparison $^{\mathrm{a}}$} & \multicolumn{2}{|c|}{ FLAIR } & \multirow[b]{2}{*}{ Relative comparison $^{\mathrm{a}}$} & \multicolumn{2}{|l|}{ DIR } & \multirow[b]{2}{*}{ Relative comparison $^{\mathrm{a}}$} \\
\hline & $1.5 \mathrm{~T}$ & $3 \mathrm{~T}$ & & $1.5 \mathrm{~T}$ & $3 \mathrm{~T}$ & & $1.5 \mathrm{~T}$ & $3 \mathrm{~T}$ & \\
\hline Intracortical & 7 & 5 & -29 & 10 & 13 & +30 & 26 & 76 & +192 \\
\hline Mixed GM-WM & 44 & 50 & +14 & 70 & 84 & +20 & 76 & 99 & +30 \\
\hline Deep GM & 3 & 4 & +33 & 3 & 4 & +33 & 5 & 5 & 0 \\
\hline Juxtacortical & 350 & 389 & +11 & 381 & 449 & +18 & 286 & 354 & +24 \\
\hline Deep WM & 448 & 513 & +15 & 516 & 609 & +18 & 408 & 518 & +27 \\
\hline Periventricular & 342 & 392 & +15 & 424 & 468 & +10 & 434 & 464 & +7 \\
\hline Infratentorial & 64 & 64 & 0 & 46 & 75 & +63 & 77 & 121 & +57 \\
\hline Total & 1,258 & 1,417 & +13 & 1,450 & 1,702 & +19 & 1,312 & 1,637 & +25 \\
\hline
\end{tabular}

${ }^{a}$ Data represent relative differences in the numbers of lesions detected at $3 \mathrm{~T}$ vs $1.5 \mathrm{~T}$ expressed as percentages

with a slice thickness according to current imaging guidelines and obtained concordant results. Using 3-T MRI, we were able to identify significantly more lesions than at $1.5 \mathrm{~T}$ in the periventricular white matter on the $\mathrm{T} 2$ TSE and additionally in the juxtacortical and infratentorial white matter on the FLAIR sequence. The DIR provided the highest lesion load measurement within the posterior fossa at both field strengths. Beside diagnostic imaging criteria, this finding is of special prognostic interest, as it has been shown that the number of infratentorial lesions at baseline plays an important prognostic role in the prediction of long-term disability in patients presenting with CIS suggestive of MS [19].

MS has been traditionally considered as a chronic inflammatory disease predominantly affecting the white matter of the CNS, which is somehow reflected by the high sensitivity of MRI in the detection of focal and diffuse white matter abnormalities in MS patients. However, pathological studies have convincingly shown that cortical grey matter lesions occur abundantly in MS across all different disease courses and are linked to certain clinical manifestations such as physical disability, cognitive impairment and epilepsy [3, 20, 21]. From the radiological point of view, grey matter manifestations in MS have been disregarded for a long time because of the low sensitivity of conventional MRI techniques in the detection of cortical lesions. As it has been shown that DIR sequences, simultaneously suppressing CSF and the white matter, are able to increase the detectability of grey matter lesions in MS significantly [13], the possible role of MRI in detecting grey matter changes in MS has become a major focus of interest. Currently, DIR applications at $1.5 \mathrm{~T}$ are increasingly being used to detect and quantify cortical lesion load in MS patients as well as to correlate these measures with the clinical course, outcome measures and symptom manifestations [14, 22-23]. Compared with

Table 3 Patient-based analysis. Data represent the number of patients with more lesions detected at $1.5 \mathrm{~T}$ or $3 \mathrm{~T}$ respectively categorised according to different anatomical regions

\begin{tabular}{|c|c|c|c|c|c|c|c|c|c|}
\hline \multirow[b]{2}{*}{ Region } & \multicolumn{2}{|l|}{$\mathrm{T} 2$} & \multirow[b]{2}{*}{$\begin{array}{l}p \\
\text { value }^{\mathrm{a}}\end{array}$} & \multicolumn{2}{|l|}{ FLAIR } & \multirow[b]{2}{*}{$\begin{array}{l}p \\
\text { value }^{\mathrm{a}}\end{array}$} & \multicolumn{2}{|l|}{$\underline{\text { DIR }}$} & \multirow[b]{2}{*}{$\begin{array}{l}p \\
\text { value }^{\mathrm{a}}\end{array}$} \\
\hline & $\begin{array}{l}\text { More lesions at } \\
1.5 \mathrm{~T}\end{array}$ & $\begin{array}{l}\text { More lesions } \\
\text { at } 3 \mathrm{~T}\end{array}$ & & $\begin{array}{l}\text { More lesions at } \\
1.5 \mathrm{~T}\end{array}$ & $\begin{array}{l}\text { More lesions } \\
\text { at } 3 \mathrm{~T}\end{array}$ & & $\begin{array}{l}\text { More lesions at } \\
1.5 \mathrm{~T}\end{array}$ & $\begin{array}{l}\text { More lesions } \\
\text { at } 3 \mathrm{~T}\end{array}$ & \\
\hline Intracortical & 4 & 1 & 0.750 & 3 & 5 & 0.563 & 0 & 26 & $<0.001$ \\
\hline $\begin{array}{l}\text { Mixed GM- } \\
\text { WM }\end{array}$ & 6 & 8 & 0.540 & 6 & 14 & 0.483 & 2 & 14 & 0.008 \\
\hline Deep GM & 2 & 3 & 1.000 & 3 & 3 & 1.000 & 2 & 2 & 1.000 \\
\hline Juxtacortical & 10 & 16 & 0.136 & 6 & 21 & 0.005 & 5 & 20 & 0.001 \\
\hline Deep WM & 6 & 21 & 0.002 & 5 & 23 & $<0.001$ & 3 & 24 & $<0.001$ \\
\hline Periventricular & 9 & 17 & 0.016 & 8 & 18 & 0.027 & 9 & 17 & 0.060 \\
\hline Infratentorial & 9 & 7 & 0.993 & 3 & 11 & 0.012 & 1 & 20 & $<0.001$ \\
\hline Total & 9 & 20 & 0.002 & 5 & 27 & $<0.001$ & 3 & 30 & $<0.001$ \\
\hline
\end{tabular}

${ }^{\text {a }} P$ value obtained by Wilcoxon's test for matched pairs. $P$ values $<0.05$ are considered statistically significant 
Fig. 2 Transverse sections of the supratentorial brain of a 31-year-old man with relapsingremitting MS. Upper row images were obtained at $3 \mathrm{~T}$, bottom row images were obtained at 1.5 T. Left column DIR images, middle column FLAIR images, right column T2 TSE images. The 3-T images offer better image quality allowing better delineation of focal inflammatory lesions, regardless of the specific anatomical location. At both field strengths, the DIR sequence allows a better classification of lesions close to the cortex or in the cortex itself as juxtacortical lesions (closedhead arrows) or cortical lesions and mixed white matter-grey matter lesions (open-head arrows), respectively. In particular, the DIR at $3 \mathrm{~T}$ shows more lesions purely located in the grey matter and lesions partially located in the grey and white matter

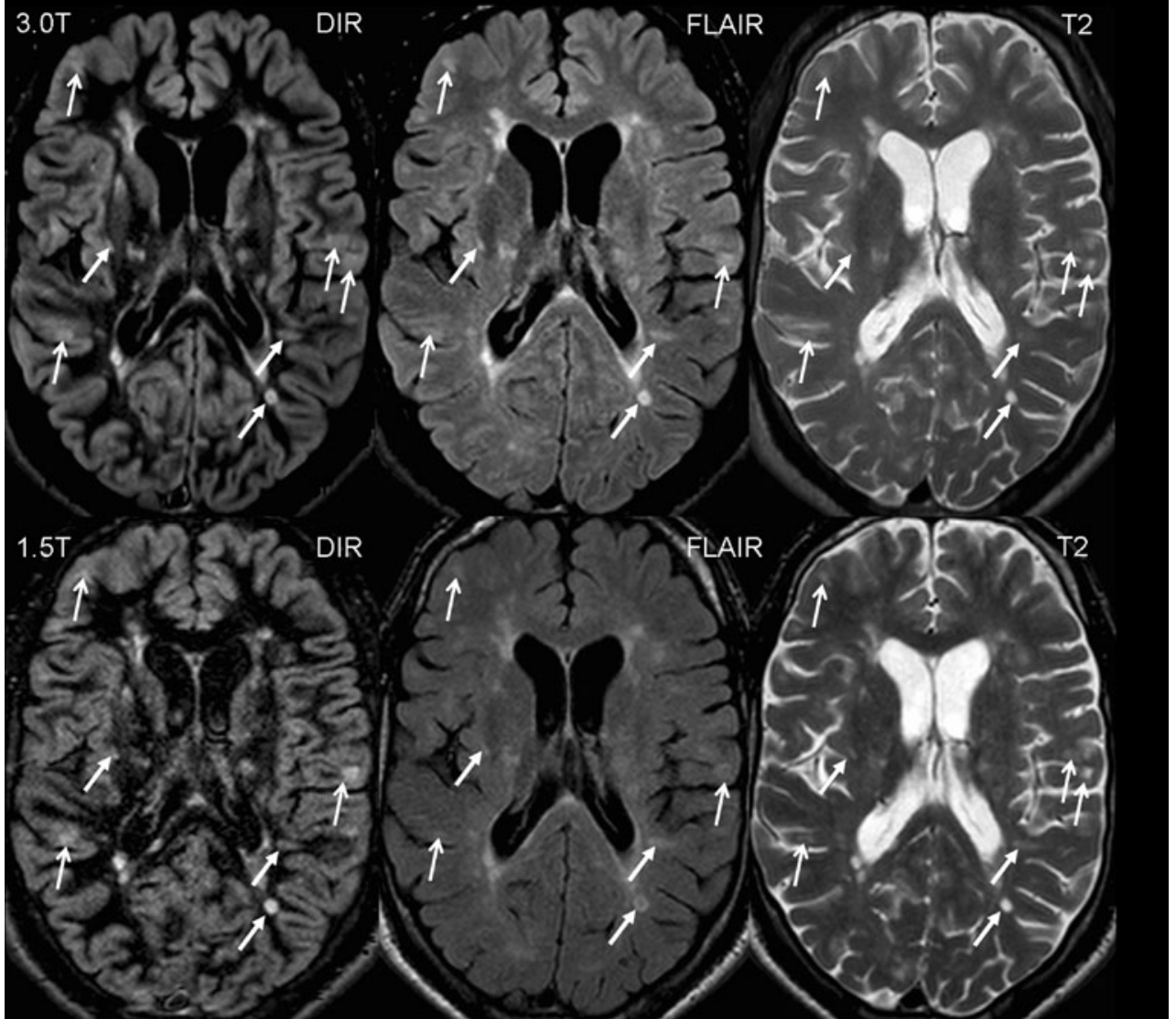

standard pulse sequences such as FLAIR and T2 TSE, substantial drawbacks of DIR applications at $1.5 \mathrm{~T}$ are the higher propensity to produce artefacts (in particular because of vessel and CSF flow) but also the relatively low SNR due to the application of two consecutive inversion pulses. These factors can substantially impair the image quality and diagnostic accuracy in many disease entities [24-26]. Because of the higher SNR, imaging at higher magnetic field strengths is, therefore, a promising application of DIR imaging, allowing a further characterisation of cortical structures even with a high spatial resolution [27].

In agreement with recent studies at $1.5 \mathrm{~T}$ and $3 \mathrm{~T}$, our study showed that DIR is of greater benefit in the detection of pure intracortical and mixed white matter-grey matter lesions compared with FLAIR or conventional T2weighted TSE sequences at both field strengths. This finding is mainly based on two different observations: on a better contrast between lesions and the surrounding normal-appearing grey matter due to a slight attenuation of the cortex itself on DIR [13], and also because of a better distinction between pure cortical, mixed white matter-grey matter and juxtacortical lesions on DIR images [13, 28]. Many of these lesions are scored as juxtacortical on conventional T2-weighted FLAIR images leading to a high amount of juxtacortical lesions and rather low numbers of cortical lesions [29]. This explains the relatively lower numbers of juxtacortical lesions on DIR images (regardless of the field strength used) because many of these lesions were classified as pure cortical or mixed white matter-grey matter lesions.

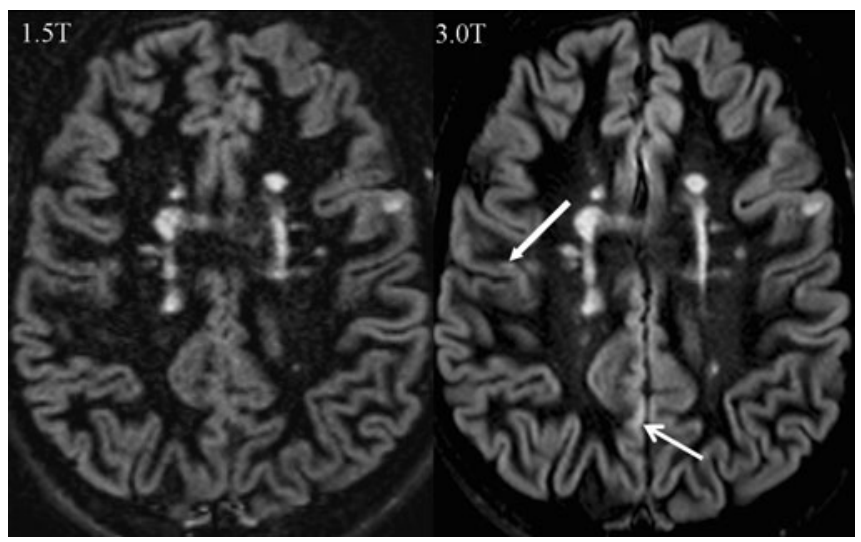

Fig. 3 Transversal sections of a DIR sequence at $1.5 \mathrm{~T}$ (left) and $3 \mathrm{~T}$ (right) from a 44-year-old woman with relapsing-remitting MS. Please note the higher conspicuity and improved detection of a lesion purely located in the cortex (closed-head arrow). Please note also the diffuse high signal changes in the parasagittal cingulate cortex which can also be frequently observed in healthy control subjects and have to be considered artefacts (open-head arrow) 


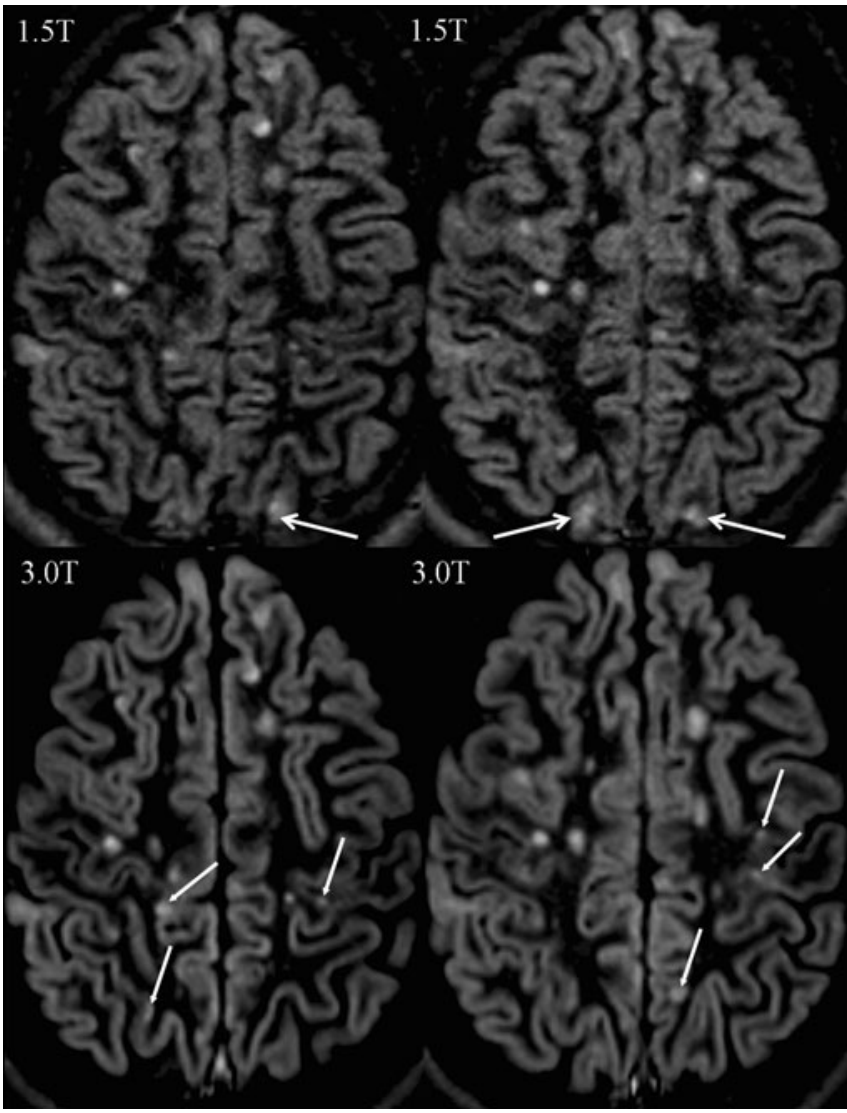

Fig. 4 Two consecutive DIR sections of the supratentorial brain of a 31-year-old man with relapsing-remitting MS obtained at $1.5 \mathrm{~T}$ (top row) and $3 \mathrm{~T}$ (bottom row). The DIR sequence benefits from the higher signal at $3 \mathrm{~T}$ leading to better delineation of the cortex and inflammatory lesions affecting the cortex (close-headed arrows). Please note also diffuse symmetric high signal changes in the occipital cortex (open-head arrows) which do not represent real cortical abnormalities and have to be considered artefacts

Moving to higher magnetic field strengths, the results of our studies have shown that 3-T MRI allows a significantly higher in vivo lesion load measurement of cortical lesions in MS patients compared with $1.5 \mathrm{~T}$. However, this higher sensitivity in the detection of cortical lesions using $3 \mathrm{~T}$ instead of $1.5 \mathrm{~T}$ was only present on DIR and was not significant for the T2-weighted or FLAIR sequences. DIR was the only sequence for which $3 \mathrm{~T}$ showed a significant increase in both pure cortical and mixed white matter-grey matter lesions. Keeping in mind that cortical lesions correlate strongly with clinical outcome measures such as cognitive impairment and physical disability $[14,20]$, the clinical relevance of higher in vivo cortical lesion load measurement using clinical highfield MRI operating at $3 \mathrm{~T}$ becomes even more obvious.

The higher cortical lesion load at 3-T MRI using DIR is probably based on a higher SNR and on the re-classifica- tion of juxtacortical lesions into pure cortical or mixed white matter-grey matter lesions. This can, of course, have substantial consequences in terms of classification of patients (particularly CIS patients) according to MRI criteria [5-7]. As 3-T MRI systems are increasingly being used in a clinical routine setting and multi-centre treatment trials, this higher lesion load of cortical lesions at $3 \mathrm{~T}$ and the substantial clinical relevance of cortical lesions are good arguments when considering cortical lesions for future imaging and diagnostic criteria.

However, compared with histopathological studies showing up to nearly $60 \%$ of the lesion load in MS patients to be located in cortical areas, we have to be aware that the sensitivity of MRI using DIR even at $3 \mathrm{~T}$ remains relatively rather low. Only $10 \%$ of all detected lesions on DIR at $3 \mathrm{~T}$ were (at least partially) located in the cortex.

The next step might be to investigate whether the next generation of whole-body high-field MRI operating at $7 \mathrm{~T}$ can further increase the in vivo detection of cortical lesions. First results are promising but also challenging in particular with regard to the implementation of DIR imaging techniques [30-33].

A limitation of our study is that our image analysis of cortical lesions was limited to the classification of pure cortical and combined (mixed) grey matter-white matter lesions. Although we applied a spatial resolution according to current MS imaging guidelines, we did not try to further classify cortical lesions according to histopathological cortical lesion-type classification. In particular, subpial demyelination, which is the most frequent type of lesion manifestation in the cortex, cannot be reliably visualised on MRI even using ultra-high-field MRI because of a lack of myelin in the superficial cortical layers and a lack of inflammation [34, 35]. Moreover, our study is based on two-dimensional (2D) pulse sequences. The implementation of 3D sequences allowing the acquisition of highresolution imaging with isotropic voxels and reducing flow artefacts might further increase the lesion count of cortical lesions [13, 36-37]. Additionally, we have to be aware that the readers might not have been sufficiently blinded to the images in terms of the magnetic field strength. Although the readers were formally blinded to the magnetic field strengths, they might have been able identify the 3-T and 1.5-T image by analysing the better image quality and lesion conspicuity on $3 \mathrm{~T}$. This could have resulted in a certain reading bias, which cannot be avoided, however, and is a limitation for all intra-individual magnetic field strength comparisons.

In conclusion, the combination of 3-T MRI and a dedicated DIR pulse sequence leads to a significantly higher sensitivity for the detection of cortical lesions in MS patients, which might lead to considerable consequences for future imaging studies dealing with clinical outcome measures and diagnostic criteria. 
Acknowledgements The authors would like to thank all participants who agreed to take part in this study. Carsten Lukas was supported by a fellowship grant provided by Bayer Schering AG, Berlin, Germany. The Amsterdam MS Center is supported by the Dutch Foundation for MS Research (Voorschoten, The Netherlands).
Open Access This article is distributed under the terms of the Creative Commons Attribution Noncommercial License which permits any noncommercial use, distribution, and reproduction in any medium, provided the original author(s) and source are credited.

\section{References}

1. Bakshi R, Thompson AJ, Rocca MA et al (2008) MRI in multiple sclerosis: current status and future prospects. Lancet Neurol 7:615-625

2. Polman $\mathrm{CH}$, Reingold SC, Edan $\mathrm{G}$ et al (2005) Diagnostic criteria for multiple sclerosis: 2005 revisions to the "McDonald Criteria". Ann Neurol 58:840-846

3. Kidd D, Barkhof F, McConnell R, Algra PR, Allen IV, Revesz T (1999) Cortical lesions in multiple sclerosis. Brain 122:17-26

4. Geurts JJG, Barkhof F (2008) Grey matter pathology in multiple sclerosis. Lancet Neurol 7:841-851

5. Barkhof F, Filippi M, Miller DH et al (1997) Comparison of MRI criteria at first presentation to predict conversion to clinically definite multiple sclerosis. Brain 120:2059-2069

6. Tintoré M, Rovira A, Martinez MJ et al (2000) Isolated demyelinating syndromes: comparison of different MR imaging criteria to predict conversion to clinically definite multiple sclerosis. AJNR Am J Neuroradiol 21:702-706

7. Swanton JK, Rovira A, Tintore A et al (2007) MRI criteria for multiple sclerosis in patients with clinically isolated syndromes: a multicentre retrospective study. Lancet Neurol 6:677-686

8. Geurts JJG, Bo L, Pouwels PJW, Castelijns JA, Polman $\mathrm{CH}$, Barkhof $\mathrm{F}$ (2005) Cortical lesions in multiple sclerosis: combined postmortem MR imaging and histopathology. AJNR Am J Neuroradiol 26:572-577

9. Kuhl CK, Träber F, Schild HH (2008) Whole-body high-field-strength (3.0-T) MR imaging in clinical practice. Part I. Technical considerations and clinical applications. Radiology 246:675-696

10. Wattjes MP, Barkhof F (2009) High field MRI in the diagnosis of MS: high field-high yield? Neuroradiology 51:279-292

11. Kurtzke JF (1983) Rating neurologic impairment in multiple sclerosis: an expanded disability status scale (EDSS). Neurology 33:1444-1452
12. Simon JH, Li D, Traboulsee A et al (2006) Standardized MR imaging protocol for multiple sclerosis: consortium of MS centers consensus guidelines. AJNR Am J Neuroradiol 27:455-461

13. Geurts JJG, Pouwels PJW, Uitdehaag BMJ, Polman CH, Barkhof F, Castelijns JA (2005) Intracortical lesions in multiple sclerosis: improved detection with double inversion-recovery MR imaging. Radiology 236:254-260

14. Calabrese M, De Stefano N, Atzori M et al (2007) Detection of cortical inflammatory lesions by double inversion recovery magnetic resonance imaging in patients with multiple sclerosis. Arch Neurol 64:1416-1422

15. Tan IL, Pouwels PJ, van Schijndel RA, Ader HJ, Manoliu RA, Barkhof F (2002) Isotropic 3D fast FLAIR imaging of the brain in multiple sclerosis patients: initial experience. Eur Radiol 12:559-567

16. Di Perry C, Dwyer MG, Wack DS et al (2009) Signal abnormalities on 1.5 and 3 Tesla brain MRI in multiple sclerosis patients and healthy controls. A morphological and spatial quantitative comparison study. Neuroimage 47:1352-1362

17. Wattjes MP, Lutterbey GG, Harzheim $M$ et al (2006) Higher sensitivity in the detection of inflammatory brain lesions in patients with clinically isolated syndromes suggestive of multiple sclerosis using high field MRI: an intraindividual comparison of $1.5 \mathrm{~T}$ with 3.0T. Eur Radiol 16:2067-2073

18. Wattjes MP, Harzheim M, Kuhl CK et al (2006) Does high-field MRI have an influence on the classification of patients with clinically isolated syndromes according to current diagnostic magnetic resonance imaging criteria for multiple sclerosis? AJNR Am J Neuroradiol 27:1794-1798

19. Minneboo A, Barkhof F, Polman CH et al (2004) Infratentorial lesions predict long-term disability in patients with initial findings suggestive of multiple sclerosis. Arch Neurol 61:217-221

20. Rovaris M, Filippi M, Minicucci L et al (2000) Cortical/subcortical disease burden and cognitive impairment in multiple sclerosis. AJNR Am J Neuroradiol 21:402-408
21. Spatt J, Chaix R, Mamoli B (2001) Epileptic and non-epileptic seizures in multiple sclerosis. J Neurol 248:2-9

22. Calabrese M, De Stefano N, Atzori M et al (2008) Extensive cortical inflammation is associated with epilepsy in multiple sclerosis. J Neurol 255:581586

23. Calabrese M, Rocca MA, Atzori M et al (2009) Cortical lesions in primary progressive multiple sclerosis: a 2-year longitudinal MR study. Neurology 72:1330-1336

24. Redpath TW, Smith FW (1994) Technical note: use of a double inversion recovery pulse sequence to image selectively grey or white brain matter. Br J Radiol 67:1258-1263

25. Bedell BJ, Narayana PA (1998) Implementation and evaluation of a new pulse sequence for rapid acquisition of double inversion recovery images for simultaneous suppression of white matter and CSF. J Magn Reson Imaging 8:544-547

26. Turetschek K, Wunderbaldinger $P$, Bankier AA et al (1998) Double inversion recovery imaging of the brain: initial experience and comparison with fluid attenuated inversion recovery imaging. Magn Reson Imaging 16:127135

27. Kim EY, Kim DH, Chang JH, Yoo E, Lee JW, Park HJ (2009) Triple layer appearance of Brodmann area 4 at thinsection double inversion recovery MR imaging. Radiology 250:515-522

28. Wattjes MP, Lutterbey GG, Gieseke J et al (2007) Double inversion-recovery brain imaging at 3.0 Tesla: diagnostic value in the detection of multiple sclerosis lesions. AJNR Am J Neuroradiol 28:54-59

29. Wattjes MP, Lutterbey GG, Harzheim $M$ et al (2006) Imaging of inflammatory lesions at 3.0 Tesla in patients with clinically isolated syndromes suggestive of multiple sclerosis: a comparison of fluid-attenuated inversion recovery with T2 turbo spin-echo. Eur Radiol 16:1494-1500 
30. Kollia K, Maderwald S, Putzki N et al (2009) First clinical study on ultra-high field MR imaging in patients with multiple sclerosis: comparison of $1.5 \mathrm{~T}$ and 7T. AJNR Am J Neuroradiol 30:699-702

31. Hammond KE, Metcalf M, Carvajal L et al (2008) Quantitative in vivo magnetic resonance imaging of multiple sclerosis at 7 Tesla with sensitivity to iron. Ann Neurol 64:707-713
32. Madelin G, Oesingmann N, Inglese M (2010) Double inversion recovery MRI with fat suppression at 7 Tesla: initial experience. J Neuroimaging 20:87-92 doi:10.1111/j.1552-6569.2008.00331.x

33. Mainero C, Benner T, Radding A et al (2009) In vivo imaging of cortical pathology in multiple sclerosis using ultra-high field MRI. Neurology 73:941-948

34. Bø L, Vedeler CA, Nyland H, Trapp BD, Mörk SJ (2003) Intracortical multiple sclerosis lesions are not associated with increased lymphocyte infiltration. Mult Scler 9:323-331

35. Bø L (2009) The histopathology of grey matter demyelination in multiple sclerosis. Acta Neurol Scand 120(Suppl 189):51-57
36. Moraal B, Rosendaal SD, Pouwels PJ et al (2008) Multi-contrast isotropic, single-slab 3D MR imaging in multiple sclerosis. Eur Radiol 18:2311-2320

37. Bagnato F, Butman JA, Gupta $S$ et al (2006) In vivo detection of cortical plaques by MR imaging in patients with multiple sclerosis. AJNR Am J Neuroradiol 27:2161-2167 